\title{
Observation of the Second Triatomic Resonance in Efimov's Scenario
}

\author{
Bo Huang (黄博), ${ }^{1}$ Leonid A. Sidorenkov, ${ }^{1,2}$ and Rudolf Grimm ${ }^{1,2}$ \\ ${ }^{1}$ Institut für Experimentalphysik, Universität Innsbruck, 6020 Innsbruck, Austria \\ ${ }^{2}$ Institut für Quantenoptik und Quanteninformation (IQOQI), Österreichische Akademie der Wissenschaften, 6020 Innsbruck, Austria
}

Jeremy M. Hutson

Joint Quantum Centre (JQC) Durham/Newcastle, Department of Chemistry,

Durham University, South Road, Durham DH1 3LE, United Kingdom

(Received 26 February 2014; published 12 May 2014)

\begin{abstract}
We report the observation of a three-body recombination resonance in an ultracold gas of cesium atoms at a very large negative value of the $s$-wave scattering length. The resonance is identified as the second triatomic Efimov resonance, which corresponds to the situation where the first excited Efimov state appears at the threshold of three free atoms. This observation, together with a finite-temperature analysis and the known first resonance, allows the most accurate demonstration to date of the discrete scaling behavior at the heart of Efimov physics. For the system of three identical bosons, we obtain a scaling factor of 21.0(1.3), close to the ideal value of 22.7 .
\end{abstract}

DOI: 10.1103/PhysRevLett.112.190401

PACS numbers: 34.50.Cx, 03.75.-b, 21.45.-v, 67.85.-d

Efimov's prediction of weakly bound three-body states in a system of three resonantly interacting bosons [1,2] is widely known as the paradigm of universal few-body quantum physics. Its bizarre and counterintuitive properties have attracted a great deal of attention. Originally predicted in the context of nuclear systems, Efimov states are now challenging atomic and molecular physics and have strong links to quantum many-body physics [3]. Experimentally, the famous scenario remained elusive until experiments in an ultracold gas of Cs atoms revealed the first signatures of the exotic three-body states [4]. A key requirement for the experiments is the precise control of two-body interactions enabled by magnetically tuned Feshbach resonances [5]. With advances in various atomic systems [6-17] and theoretical progress in understanding Efimov states and related states in real systems $[3,18]$, the research field of few-body physics with ultracold atoms has emerged.

Three-body recombination resonances [19] are the most prominent signatures of Efimov states [2,20]. They emerge when an Efimov state couples to the threshold of free atoms at distinct negative values of the $s$-wave scattering length $a$. The resonance positions $a_{-}^{(n)}$ are predicted to reflect the discrete scaling law at the heart of Efimov physics, and for the system of three identical bosons follow $a_{-}^{(n)}=22.7^{n} a_{-}^{(0)}$. Here, $n=0$ refers to the Efimov ground state and $n=1,2, \ldots$ refer to excited states. The starting point $a_{-}^{(0)}$ of the infinite series, i.e., the position of the ground-state resonance, is commonly referred to as the three-body parameter [16,21-24].

For an observation of the second Efimov resonance, the requirements are much more demanding than for the first one. Extremely large values of the scattering length near $a_{-}^{(1)}$ need to be controlled and the relevant energy scale is lower by a factor $22.7^{2} \approx 500$, which requires temperatures in the range of a few $\mathrm{nK}$. So far, experimental evidence for an excited-state Efimov resonance has been obtained only in a three-component Fermi gas of ${ }^{6} \mathrm{Li}$ [11], but there the scenario is more complex because of the involvement of three different scattering lengths. Experiments on bosonic ${ }^{7} \mathrm{Li}$ have approached suitable conditions for a three-boson system $[7,25,26]$ and suggest the possibility of observing the excited-state Efimov resonance [26].

In this Letter, we report on the observation of the second triatomic resonance in Efimov's original three-boson scenario realized with cesium atoms. Our results confirm the existence of the first excited three-body state and allow the currently most accurate test of the Efimov period. Moreover, our results provide evidence for the existence of the predicted universal $\mathrm{N}$-body states that are linked to the excited three-body state.

Two recent advances have prepared the ground for our present investigations. First, we have gained control of very large values of the scattering length (up to a few times $10^{5} a_{0}$ with $a_{0}$ being Bohr's radius), which in ultracold Cs gases is achieved by exploiting a broad Feshbach resonance near $800 \mathrm{G}[21,27]$. Precise values for the scattering length as a function of the magnetic field can be obtained from coupled-channel calculations based on the M2012 model potentials of Refs. [21,28]. Second, Ref. [26] has provided a model, based on an $S$-matrix formalism $[29,30]$, to describe quantitatively the finite-temperature effects on three-body recombination near Efimov resonances. While for the first Efimov resonance experimental conditions can be realized practically in the zero-temperature limit, finite-temperature limitations are unavoidable for the second resonance and therefore must be properly taken into account. 
Our experimental procedure of preparing an ultracold sample of cesium atoms near quantum degeneracy is similar to the one reported in Refs. [21,31]. In an additional stage, introduced into our setup for the present work, we adiabatically expand the atomic cloud into a very weak trap. The latter is a hybrid with optical confinement by a single infrared laser beam and magnetic confinement provided by the curvature of the magnetic field [32]. The mean oscillation frequency $\bar{\omega} / 2 \pi$ of the nearly isotropic trap is about $2.6 \mathrm{~Hz}$. This very low value corresponds to a harmonic oscillator length of $\sim 5 \mu \mathrm{m}$, which is about a factor of 5 larger than the expected size of the second Efimov state. Our ultracold atomic sample consists of about $N=3 \times 10^{4} \mathrm{Cs}$ atoms at a temperature of $7 \mathrm{nK}$ and a dimensionless phase-space density of about 0.2 . We probe the atomic cloud by in situ absorption imaging near the zero crossing of the scattering length at $882 \mathrm{G}$. We obtain the in-trap density profile and the temperature $T$ assuming the gas is thermalized in a harmonic trap.

To study recombinative decay for different values of the scattering length $a$, we ramp the magnetic field from the final preparation field $(\sim 820 \mathrm{G})$ [32] down to a target value (between 818 and $787 \mathrm{G}$ ) [33] within $10 \mathrm{~ms}$. After a variable hold time $t$, between tens of milliseconds and several seconds, we image the remaining atoms. The maximum hold time is chosen to correspond to an atom number decay of about $50 \%$. In addition to the resulting decay curves $N(t)$ we record the corresponding temperature evolution $T(t)$. Recombinative decay is known to be accompanied by heating [32,34], which needs to be taken into account when analyzing the results.

For extracting recombination rate coefficients from the observed decay curves, we apply a model that is based on the general differential equation for $\alpha$-body loss in a harmonically trapped thermal gas,

$$
\frac{\dot{N}}{N}=-L_{\alpha} \alpha^{-3 / 2}\left(\frac{N}{V}\right)^{\alpha-1},
$$

with the volume $V=\left(2 \pi k_{B} T / m \bar{\omega}^{2}\right)^{3 / 2}$. The factor $\alpha^{-3 / 2}$ arises from the spatial integration of the density-dependent losses.

Since three-body recombination is expected to dominate the decay, we fix $\alpha$ to a value of 3, numerically integrate Eq. (1) over time and fit the measured atomic number evolution with $L_{3}$ and the initial atom number $N_{0}$ as free parameters. In cases where there are significant contributions from higher-order decay processes, e.g., four-body decay, the fitted $L_{3}$ can be interpreted as an "effective" loss coefficient [35] that includes all loss processes. Considering a typical temperature change of about $50 \%$ during the decay, a slight complication arises from the fact that $L_{3}$ itself generally depends on $T$, while our fit assumes constant $L_{3}$. To a good approximation, however, we can refer a fit value for $L_{3}$ to a time-averaged temperature $T_{\text {avg }}$ [32] .

Figure 1(a) shows our main result, the recombination resonance caused by an excited Efimov state. Here we plot the fit values obtained for $L_{3}$ as a function of the inverse
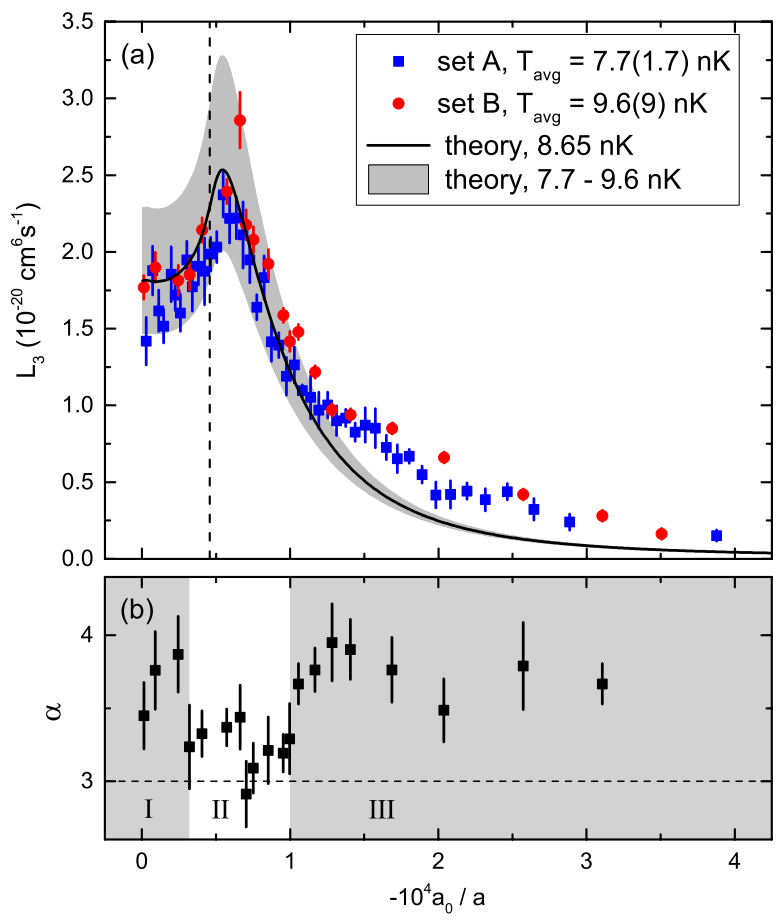

FIG. 1 (color online). Observation of the second triatomic Efimov resonance. In (a) we show the effective three-body loss coefficient $L_{3}$ as a function of the inverse scattering length. Blue squares and red circles are two sets of measurements taken with slightly different trap settings. The error bars include statistical uncertainties from numerical fitting. The black solid line is the theoretical calculation of $L_{3}$ (based on the parameters of the first Efimov resonance) at $8.65 \mathrm{nK}$ (average temperature of two sets) while the gray-shaded region corresponds to the temperature range between 7.7 and $9.6 \mathrm{nK}$ (see text). The vertical dashed line indicates the position where the resonance would be expected in the zero-temperature limit based on the previously investigated first Efimov resonance [21] and Efimov's scaling factor. In (b), the fitted loss index $\alpha$ shows a larger deviation from a value of 3 in the regions (gray-shaded) away from the second Efimov resonance (white region, where $\alpha<3.5$ ), indicating contributions from higher-order recombination processes.

scattering length $1 / a$. Our sets of measurements $(A$, blue squares and $B$, red circles) [32] were taken on different days with similar trap frequencies but slightly different average temperatures $T_{\text {avg }}$ of 7.7(1.7) and 9.6(9) nK [32]. Our results exhibit a loss peak near $a=-17000 a_{0}(\sim 797 \mathrm{G})$, which we interpret as a clear manifestation of the second Efimov resonance. Multiplying $a_{-}^{(0)}=-963 a_{0}$ [32] by Efimov's ideal scaling factor of 22.7 predicts that, in the zerotemperature limit, this feature would occur at $-21900 a_{0}$ [dashed vertical line in Fig. 1(a)]. At finite temperatures, however, a down-shift towards somewhat lower values of $|a|$ is expected [4] and may to a large extent explain the observed position. The finite temperature in our experiment also explains why the resonance is not as pronounced as the first Efimov resonance observed previously [21].

In order to compare our results with theoretical predictions, we use the finite-temperature model of Ref. [26] 
with the two resonance parameters, position $a_{-}^{(0)}=-963 a_{0}$ and decay parameter $\eta_{-}^{(0)}=0.10$ [32], independently derived from previous measurements on the first Efimov resonance. For the temperature we use $T_{\mathrm{avg}}=8.65 \mathrm{nK}$, which is the mean value for the two sets. The agreement between our present results and the prediction [black solid line in Fig. 1(a)] is remarkable, and highlights the discrete scaling behavior of the Efimov scenario.

The measurements on the "shoulder" of the resonance $\left[-10^{4} a_{0} / a>1.2\right.$ in Fig. 1(a)] show a broad increase of the effective $L_{3}$ as compared to the expectation from the threebody loss theory [black solid line in Fig. 1(a)]. Since similar enhanced loss features were observed previously near the first Efimov resonance [7,25,36-38] and were explained by the presence of four- or five-body states associated with an Efimov state, we attribute this feature to higher-order decay processes. To check this, we fit set $B$ [39] with Eq. (1) as discussed above, while now using $\alpha$ as an additional free parameter. The fit results for $\alpha$ are shown in Fig. 1(b). In the region close to the Efimov resonance (white region II), where we expect dominant three-body behavior, the value of $\alpha$ is relatively close to 3 [40]. On the shoulder of the Efimov peak (gray-shaded region III), a significant increase of $\alpha$, compared to the resonance region, confirms the existence of higher-order decay processes. It is interesting to note that the relatively broad shoulder that we observe for the higher-order features is in contrast to the narrow features observed in ${ }^{7} \mathrm{Li}[7,25]$. On the other side of the Efimov resonance (gray-shaded region I), we also observe an enhancement of $\alpha$, which is likely to be caused by similar higher-order decay features associated with highly excited $N$-body cluster states.

The temperature uncertainty plays an important role in the interpretation of our results. The measured values of $L_{3}$ depend sensitively on the temperature, with a general scaling $\propto T^{3}$ according to the volume $V$ in Eq. (1). The theoretical $L_{3}$ values also depend strongly on the temperature. The gray-shaded area in Fig. 1(a) demonstrates the variation between 7.7 and $9.6 \mathrm{nK}$, which correspond to $T_{\text {avg }}$ for sets $A$ and $B$, respectively. It may be seen that the temperature uncertainty results mainly in an amplitude error rather than an error in the peak position.

To analyze the observed resonance in more detail, and especially to study the possible small deviation of $a_{-}^{(1)}$ from a predicted value of $22.7 a_{-}^{(0)}$, we now fit the results in the resonance region $\left(0<-10^{4} a_{0} / a<1.2\right.$ in Fig. 2$)$ with the finite-temperature model to extract an experimental value for $a_{-}^{(1)}$. Here, because of the large effect of the temperature uncertainty, we use the temperature $T$ as an additional parameter in the fits. The results (blue dashed and red dotted lines in Fig. 2 for sets $A$ and $B$ ) are summarized in the upper part of Table I and yield a mean $a_{-}^{(1)}$ value of $-20270(680) a_{0}$.

The fitted results for the temperature, 8.7(2) nK for set $A$ and $10.0(2) \mathrm{nK}$ for set $B$, are somewhat larger than the independently determined temperatures $T_{\text {avg }}$, but they are

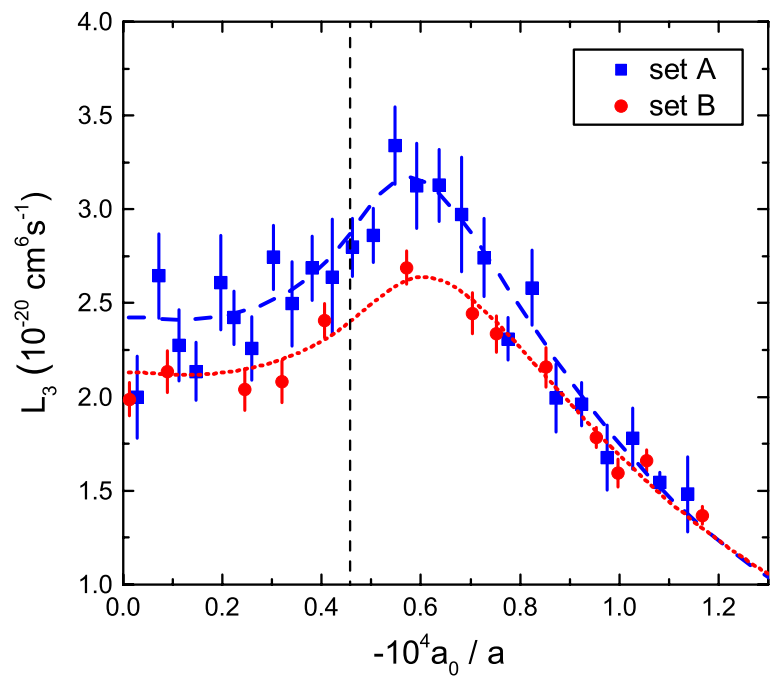

FIG. 2 (color online). Fits to the second Efimov resonance. The two sets of points represent the same sets of results as in Fig. 1, but limited to the resonance region and with one clear outlyer removed. In addition, the absolute scaling for $L_{3}$ is changed as we here use the fit values (see text) for the temperature, $8.7 \mathrm{nK}$ for set $A$ and $10.0 \mathrm{nK}$ for set $B$, to calculate the volume $V$. The vertical dashed line is the same as in Fig. 1. The fits to sets $A$ and $B$ are plotted as blue dashed and red dotted lines, respectively.

consistent with $T_{\text {avg }}$ within the error range. The higher temperatures also imply a rescaling of the measured $L_{3}$ values because of the temperature dependence of the volume $V$. With these corrections, Fig. 2 shows that the measurements of set $A$, taken at a lower temperature, now produce larger $L_{3}$ values than those of set $B$.

Uncertainties in $L_{3}$ might also arise from errors in the atom number calibration, resulting from imaging imperfections and errors in trap frequency measurements. To account for these effects, we follow an alternative fitting strategy and introduce an additional parameter $\lambda$ as an amplitude scaling factor for $L_{3}$ into the finite-temperature model, while fixing the temperature at the measured $T_{\text {avg }}$. The resulting parameters for each set are given in the lower part of Table I. Remarkably, this alternative approach gives a mean value of $-20120(630) a_{0}$ for $a_{-}^{(1)}$, which is consistent with the one extracted before. This shows the

TABLE I. Fitted parameters for the second Efimov resonance. The upper part of the Table shows the fitting results when temperature $T$ is a free parameter, while the lower part corresponds to fixed-temperature fitting with $\lambda$ as a free amplitude scaling factor. The uncertainties indicate $1 \sigma$ errors from fitting.

\begin{tabular}{lcccc}
\hline \hline Set & $T / \mathrm{nK}$ & $a_{-}^{(1)} / a_{0}$ & $\eta_{-}^{(1)}$ & $\lambda$ \\
\hline$A$ & $8.7(2)$ & $-20790(390)$ & $0.15(2)$ & $\ldots$ \\
$B$ & $10.0(2)$ & $-19740(430)$ & $0.19(3)$ & $\ldots$ \\
$A$ & $7.7^{\mathrm{a}}$ & $-20580(390)$ & $0.17(3)$ & $0.52(5)$ \\
$B$ & $9.6^{\mathrm{a}}$ & $-19650(430)$ & $0.19(3)$ & $0.80(7)$ \\
\hline
\end{tabular}

${ }^{a}$ Indicates that the corresponding parameter is kept fixed. 
robustness of our result for $a_{-}^{(1)}$. From all the four fits listed in Table I, we derive a mean value and a corresponding uncertainty of $-20190(660) a_{0}$.

A final significant contribution to our error budget for $a_{-}^{(1)}$ stems from uncertainty in the M2012 potential model $[21,28]$ that provides the mapping between the measured magnetic field $B$ and the scattering length $a$. To quantify this, we have recalculated the derivatives of all the experimental quantities fitted in Ref. [28] with respect to the potential parameters, and used them to obtain fully correlated uncertainties in the calculated scattering lengths at the magnetic fields $B=852.90$ and $795.56 \mathrm{G}$, corresponding to the two Efimov loss maxima, using the procedure of Ref. [41]. The resulting scattering lengths and their $1 \sigma$ uncertainties are $a_{-}^{(0)}=-963(6) a_{0}$ and $a_{-}^{(1)}=-20190(1000) a_{0}$. These values accord well with the uncertainty in the position of the Feshbach resonance pole, which was determined to be 786.8(6) G in Ref. [28] with a $2 \sigma$ uncertainty.

Taking all these uncertainties into account, we get $a_{-}^{(1)}=$ $-20190(1200) a_{0}$ and $a_{-}^{(0)}=-963(11) a_{0}$, and we finally obtain $a_{-}^{(1)} / a_{-}^{(0)}=21.0(1.3)$ for the Efimov period. This result is consistent with the ideal value of 22.7 within a $1.3 \sigma$ uncertainty range. Theories that take the finite interaction range into account consistently predict corrections toward somewhat lower values than 22.7 [42-44]. Reference [24] predicts a value of 17.1 in the limit of strongly entrancechannel-dominated Feshbach resonances. This theoretical value differs by $3 \sigma$ from our experimental result, but the precise value depends at a $10 \%$ level on a form factor that accounts for the range of the coupling between the open and closed channels. Universal van der Waals theory [45] applied to our specific Feshbach resonance predicts a value that is smaller than the ideal Efimov factor by only 5\%-10\% [46], which would match our observation.

Additional systematic uncertainties may slightly influence our experimental determination of the Efimov period. Model dependence in the earlier fit to various interaction-dependent observables in Cs [28] may somewhat affect the mapping $a(B)$ from magnetic field to scattering length. The finitetemperature model [26] applied here, which employs the zero-range approximation, may be influenced by small finiterange corrections. Moreover, confinement-induced effects may play an additional role even in the very weak trap $[47,48]$. While an accurate characterization of these possible systematic effects will require further effort, we estimate that our error budget is dominated by the statistical uncertainties.

Previous experiments aimed at determining the Efimov period in ${ }^{39} \mathrm{~K}[6]$ and ${ }^{7} \mathrm{Li}[7,25]$ considered recombination minima for $a>0$, from which values of 25(4) and 16.0(1.3) were extracted, respectively. There the lower recombination minima serving as lower reference points appear at quite small values of the scattering length (typically only at 3 to 4 times the van der Waals length $R_{\mathrm{vdW}}$ [5]), so that substantial quantitative deviations from Efimov's scenario, which is strictly valid only in the zero-range limit $|a| / R_{\mathrm{vdW}} \rightarrow \infty$, may be expected. In our case the lower reference point $a_{-}^{(0)}$ is at about $-9.5 R_{\mathrm{vdW}}$ (with $R_{\mathrm{vdW}}=101 a_{0}$ for Cs) [21-24], which makes the situation more robust. Moreover, at negative scattering length possible effects related to a nonuniversal behavior of the weakly bound dimer state are avoided [49]. Another difference between our work and previous determinations of the Efimov period is the character of the Feshbach resonance, which in our case is the most extreme case so far discovered of an entrance-channeldominated resonance, where the whole interaction can be reduced to an effective single-channel model [5]. The resonances exploited in ${ }^{39} \mathrm{~K}$ and ${ }^{7} \mathrm{Li}$ have intermediate character, so that the interpretation is less straightforward.

In conclusion, our observation of the second triatomic recombination resonance in an ultracold gas of Cs atoms demonstrates the existence of an excited Efimov state. Together with a previous observation of the first resonance and an analysis based on finite-temperature theory, our results provide an accurate quantitative test of Efimov's scenario of three resonantly interacting bosons. The character of the extremely broad Feshbach resonance that we use for interaction tuning avoids complications from the two-channel nature of the problem and brings the situation in a real atomic system as close as possible to Efimov's original idea. The value of 21.0(1.3) that we extract for the Efimov period is very close to the ideal value of 22.7 and represents the most accurate demonstration so far of the discrete scaling behavior at the heart of Efimov physics. Our results challenge theory to describe accurately the small deviations that occur in real atomic systems.

New possibilities for Efimov physics beyond the original three-boson scenario are opened up by ultracold mixtures with large mass imbalance [50]. The ${ }^{133}{ }^{\mathrm{Cs}}-{ }^{6} \mathrm{Li}$ mixture, where the Efimov period is reduced to a value of 4.88, has been identified as a particularly interesting system [51,52]. Two very recent preprints $[53,54]$ report the observation of consecutive Efimov resonances in this system.

We thank D. Petrov for stimulating discussions and for providing the source code for the finite-temperature model. We thank C. Salomon for important discussions and comments on the manuscript. We further thank B. Rem, Y. Wang, P. S. Julienne, J. Levinsen, R. Schmidt, and W. Zwerger for fruitful discussions and M. Berninger, A. Zenesini, H.-C. Nägerl, and F. Ferlaino for their important contributions in an earlier stage of the experiments. We acknowledge support by the Austrian Science Fund FWF within project P23106 and by EPSRC under Grant No. EP/I012044/1.

[1] V. Efimov, Phys. Lett. 33B, 563 (1970).

[2] E. Braaten and H.-W. Hammer, Phys. Rep. 428, 259 (2006).

[3] Special Issue on Efimov Physics, edited by A. S. Jensen [Few-Body Syst. 51, 77 (2011)]. 
[4] T. Kraemer, M. Mark, P. Waldburger, J. G. Danzl, C. Chin, B. Engeser, A. D. Lange, K. Pilch, A. Jaakkola, H.-C. Nägerl, and R. Grimm, Nature (London) 440, 315 (2006).

[5] C. Chin, R. Grimm, P. S. Julienne, and E. Tiesinga, Rev. Mod. Phys. 82, 1225 (2010).

[6] M. Zaccanti, B. Deissler, C. D'Errico, M. Fattori, M. JonaLasinio, S. Müller, G. Roati, M. Inguscio, and G. Modugno, Nat. Phys. 5, 586 (2009).

[7] S. E. Pollack, D. Dries, and R. G. Hulet, Science 326, 1683 (2009).

[8] N. Gross, Z. Shotan, S. Kokkelmans, and L. Khaykovich, Phys. Rev. Lett. 103, 163202 (2009).

[9] J. H. Huckans, J. R. Williams, E. L. Hazlett, R. W. Stites, and K. M. O’Hara, Phys. Rev. Lett. 102, 165302 (2009).

[10] T. B. Ottenstein, T. Lompe, M. Kohnen, A. N. Wenz, and S. Jochim, Phys. Rev. Lett. 101, 203202 (2008).

[11] J. R. Williams, E. L. Hazlett, J. H. Huckans, R. W. Stites, Y. Zhang, and K. M. O'Hara, Phys. Rev. Lett. 103, 130404 (2009).

[12] N. Gross, Z. Shotan, S. Kokkelmans, and L. Khaykovich, Phys. Rev. Lett. 105, 103203 (2010).

[13] T. Lompe, T. B. Ottenstein, F. Serwane, K. Viering, A. N. Wenz, G. Zürn, and S. Jochim, Phys. Rev. Lett. 105, 103201 (2010).

[14] S. Nakajima, M. Horikoshi, T. Mukaiyama, P. Naidon, and M. Ueda, Phys. Rev. Lett. 105, 023201 (2010).

[15] G. Barontini, C. Weber, F. Rabatti, J. Catani, G. Thalhammer, M. Inguscio, and F. Minardi, Phys. Rev. Lett. 103, 043201 (2009).

[16] S. Roy, M. Landini, A. Trenkwalder, G. Semeghini, G. Spagnolli, A. Simoni, M. Fattori, M. Inguscio, and G. Modugno, Phys. Rev. Lett. 111, 053202 (2013).

[17] R. J. Wild, P. Makotyn, J. M. Pino, E. A. Cornell, and D. S. Jin, Phys. Rev. Lett. 108, 145305 (2012).

[18] Y. Wang, J. P. D’Incao, and B. D. Esry, Adv. At. Mol. Opt. Phys. 62, 1 (2013).

[19] B. D. Esry, C. H. Greene, and J. P. Burke, Phys. Rev. Lett. 83, 1751 (1999).

[20] F. Ferlaino, A. Zenesini, M. Berninger, B. Huang, H.-C. Nägerl, and R. Grimm, Few-Body Syst. 51, 113 (2011).

[21] M. Berninger, A. Zenesini, B. Huang, W. Harm, H.-C. Nägerl, F. Ferlaino, R. Grimm, P. S. Julienne, and J. M. Hutson, Phys. Rev. Lett. 107, 120401 (2011).

[22] J. Wang, J. P. D'Incao, B. D. Esry, and C. H. Greene, Phys. Rev. Lett. 108, 263001 (2012).

[23] P. K. Sørensen, D. V. Fedorov, A. S. Jensen, and N. T. Zinner, Phys. Rev. A 86, 052516 (2012).

[24] R. Schmidt, S. Rath, and W. Zwerger, Eur. Phys. J. B 85, 386 (2012).

[25] P. Dyke, S. E. Pollack, and R. G. Hulet, Phys. Rev. A 88, 023625 (2013).

[26] B. S. Rem, A. T. Grier, I. Ferrier-Barbut, U. Eismann, T. Langen, N. Navon, L. Khaykovich, F. Werner, D. S. Petrov, F. Chevy, and C. Salomon, Phys. Rev. Lett. 110, 163202 (2013).

[27] M. D. Lee, T. Köhler, and P. S. Julienne, Phys. Rev. A 76, 012720 (2007).

[28] M. Berninger, A. Zenesini, B. Huang, W. Harm, H.-C. Nägerl, F. Ferlaino, R. Grimm, P. S. Julienne, and J. M. Hutson, Phys. Rev. A 87, 032517 (2013).

[29] V. Efimov, Sov. J. Nucl. Phys. 29, 546 (1979).
[30] E. Braaten, H.-W. Hammer, D. Kang, and L. Platter, Phys. Rev. A 78, 043605 (2008).

[31] M. Berninger, Ph.D. thesis, University of Innsbruck, 2011.

[32] See Supplemental Material at http://link.aps.org/ supplemental/10.1103/PhysRevLett.112.190401 for details on the sample preparation, the density and temperature determination, fitting of the decay curves, additional information of the two sets of results, and for an updated fit of the $L_{3}$ data obtained previously [21] for the first Efimov resonance.

[33] The magnetic field range between 787 and $818 \mathrm{G}$ corresponds to a range of scattering lengths between $-10^{5}$ and $-2600 a_{0}$.

[34] T. Weber, J. Herbig, M. Mark, H.-C. Nägerl, and R. Grimm, Phys. Rev. Lett. 91, 123201 (2003).

[35] J. von Stecher, J. P. D'Incao, and C. H. Greene, Nat. Phys. 5, 417 (2009).

[36] F. Ferlaino, S. Knoop, M. Berninger, W. Harm, J. P. D'Incao, H.-C. Nägerl, and R. Grimm, Phys. Rev. Lett. 102, 140401 (2009).

[37] A. Zenesini, B. Huang, M. Berninger, S. Besler, H.-C. Nägerl, F. Ferlaino, R. Grimm, C. H. Greene, and J. von Stecher, New J. Phys. 15, 043040 (2013).

[38] R. J. Fletcher, A. L. Gaunt, N. Navon, R. P. Smith, and Z. Hadzibabic, Phys. Rev. Lett. 111, 125303 (2013).

[39] We use only set $B$ for this purpose, because it contains more data at different hold times and therefore the fit converges better when both $L_{\alpha}$ and $\alpha$ are free in the fitting procedure.

[40] The small deviation from $\alpha=3$ in region II can be explained by the heating effect, which leads to somewhat faster decay in the initial stage and a somewhat slower decay at the end of the hold time. This mimics higher-order loss.

[41] D. L. Albritton, A. L. Schmeltekopf, and R. Zare, in Molecular Spectroscopy: Modern Research, Vol. II, edited by K. N. Rao (Academic Press, New York, 1976).

[42] J. P. D'Incao, C. H. Greene, and B. D. Esry, J. Phys. B 42, 044016 (2009).

[43] L. Platter, C. Ji, and D. R. Phillips, Phys. Rev. A 79, 022702 (2009).

[44] M. Thøgersen, D. V. Fedorov, and A. S. Jensen, Phys. Rev. A 78, 020501(R) (2008).

[45] Y. Wang and P. S. Julienne, arXiv:1404.0483.

[46] Y. Wang and P. S. Julienne (private communication).

[47] S. Jonsell, H. Heiselberg, and C. J. Pethick, Phys. Rev. Lett. 89, 250401 (2002).

[48] J. Levinsen, P. Massignan, and M. M. Parish, arXiv:1402.1859.

[49] A. Zenesini, B. Huang, M. Berninger, H.-C. Nägerl, F. Ferlaino, and R. Grimm (to be published).

[50] J. P. D'Incao and B. D. Esry, Phys. Rev. A 73, 030703(R) (2006).

[51] M. Repp, R. Pires, J. Ulmanis, R. Heck, E. D. Kuhnle, M. Weidemüller, and E. Tiemann, Phys. Rev. A 87, 010701 (2013).

[52] S.-K. Tung, C. Parker, J. Johansen, C. Chin, Y. Wang, and P. S. Julienne, Phys. Rev. A 87, 010702 (2013).

[53] S.-K. Tung, K. Jimenez-Garcia, J. Johansen, C. V. Parker, and C. Chin, arXiv:1402.5943.

[54] R. Pires, J. Ulmanis, S. Häfner, M. Repp, A. Arias, E. D. Kuhnle, and M. Weidemüller, arXiv:1403.7246. 